\title{
La pobreza monetaria desde la perspectiva de la pobreza multidimensional: el caso peruano
}

\author{
Dante A. Urbina y Milagros R. Quispe \\ Universidad de Lima, Universidad Peruana de Ciencias Aplicadas
}

Recibido: 3/7/2017 - Aprobado: 6/10/2017

\begin{abstract}
Resumen: Existen varios enfoques sobre la pobreza, pero hay dos que son más usados: la pobreza monetaria y la pobreza multidimensional. Este trabajo analiza de los determinantes de la pobreza en el Perú vinculando ambos enfoques por medio de una regresión logística en distintos periodos. Se halla que el acceso a servicios (agua, electricidad, telefonía) y la propiedad de la vivienda reducen considerablemente la probabilidad de ser pobre, y que tener seguro de salud también la reduce pero en una menor medida. Resalta el hecho de que tener educación primaria o secundaria no incide significativamente en la reducción de la probabilidad de ser pobre, lo cual puede deberse a restricciones en el actual mercado de trabajo.
\end{abstract}

Palabras clave: pobreza, acceso a servicios, derechos de propiedad, educación

\section{Monetary poverty from the perspective of multidimensional poverty: The Peruvian case}

AвstRACT: There are several approaches to poverty, but two of them are more widely used: monetary poverty and multidimensional poverty. This paper analyzes the determinants of poverty in Peru, linking both approaches by means of a logistic regression in distinct periods. It is found that access to services (water, electricity, telephony) and housing ownership reduce remarkably the probability of being poor and that having health insurance also reduces it, but to a lesser extent. Interestingly, primary or secondary education does not have a significant impact in reducing the probability of being poor, which can be due to restrictions in the current labor market.

Keywords: poverty, access to services, property rights, education 


\section{INTRODUCCIÓN}

El Perú ha experimentado un importante crecimiento económico en los últimos años. Pero la cuestión fundamental es si este crecimiento realmente ha contribuido, directa o indirectamente, a mejorar las condiciones de vida de las personas más vulnerables de nuestra sociedad, los pobres. De ahí que el combate contra la pobreza sea un tema central en la política económica de los gobiernos.

En ese contexto, los gobiernos de Alan García (2006-2011) y Ollanta Humala (2011-2016) han procurado resaltar sus acciones y resultados en materia de reducción de la pobreza. En efecto, "El Perú avanza" fue el lema del gobierno de García, mientras que Humala, con sus varios programas sociales, puso énfasis en caracterizar a su gobierno como un régimen de “inclusión social". De hecho, el 20 de octubre del 2011 se creó un ministerio específico para el efecto: el Ministerio de Desarrollo e Inclusión Social.

Ahora bien, entre el 2009 y el 2015 la pobreza en el Perú ha decrecido, pasando del 33,5 al 21,8 \%, es decir, ha experimentado una disminución de casi 12 puntos porcentuales. Se ha avanzado, ciertamente, pero aún queda mucho por hacer. La pobreza sigue siendo un problema considerable y pendiente en nuestro país. Resulta no solo pertinente sino también necesario analizar el fenómeno de la pobreza y sus determinantes en el Perú en términos de lo que han dejado los dos gobiernos precedentes.

\section{MARCO TEÓRICO}

No existe un concepto único de pobreza. De ahí que establecer una única definición de pobreza sea verdaderamente una cuestión compleja. Se puede decir que hay un cierto consenso en entender la pobreza como aquella situación en que una o más personas tienen un nivel de bienestar inferior al mínimo aceptable. Sin embargo, apenas se quiere saber qué significa exactamente "nivel de bienestar" y determinar cuál es el "mínimo aceptable", el consenso se quiebra.

Pues bien, discutir la naturaleza de la pobreza no es un ejercicio meramente teórico sino que tiene muy importantes consecuencias prácticas. $Y$ es que el concepto que se tenga de pobreza, de modo implícito o explícito, definitivamente condicionará o incluso determinará los métodos de medición de la misma. Ahora bien, establecer quién es o no es pobre determina a su vez quién deberá recibir o no ayuda de determinado programa social o sobre qué personas o grupos se focalizará determinada política. Cada 
definición de pobreza y, con ello, cada elección metodológica, incluirá a ciertas personas y dejará fuera a otras. Medidas demasiado restrictivas de la pobreza pueden hacer que se deje fuera de consideración a personas y grupos que realmente necesitan el apoyo de políticas sociales, mientras que medidas demasiado amplias pueden hacer que se asignen recursos públicos a personas que no tienen carencias tan urgentes, de modo que se podría dar un uso más eficiente a los mismos.

Hay varios enfoques sobre la pobreza. Sin embargo, hay dos en particular que son más ampliamente usados en la investigación y el diseño de políticas: el de la pobreza monetaria y el de la pobreza multidimensional.

De acuerdo con el enfoque de la pobreza monetaria, esta se puede medir a través del ingreso o del gasto. De este modo, se considera pobre monetario a aquel individuo que tiene menos que determinado ingreso por día o por mes o, alternativamente, a aquel hogar que tiene un nivel de gasto per cápita inferior al necesario para adquirir una canasta básica de consumo, la cual incluye bienes alimentarios y no alimentarios. Entonces, el aspecto clave en la medición desde este enfoque consiste en determinar la "línea de pobreza" dada por el costo de la canasta básica de consumo.

Entre las mediciones de pobreza monetaria, la más conocida es la del Banco Mundial cuya "línea de pobreza" está en USD 1,9 dólares por día a precios del 2011 (Ravallion, 2015). En cuanto al Perú, tenemos que el enfoque de pobreza monetaria es claramente el usado por el Instituto Nacional de Estadística e Informática (INEI), entidad encargada de recopilar la información sobre pobreza con base en la Encuesta Nacional de Hogares (ENAHO). En efecto, en su informe Evolución de la Pobreza Monetaria 2009-2015 dice:

La línea de pobreza es el valor monetario con el cual se contrasta el gasto per cápita mensual de un hogar para determinar si está en condiciones de pobreza o no. Este valor está conformado por dos componentes: el componente alimentario, que es llamado línea de pobreza extrema, y el componente no alimentario. El componente alimentario de la línea lo constituye el valor de una canasta socialmente aceptada de productos alimenticios. Los productos que componen esa canasta se han establecido sobre la base de los patrones de consumo real de los hogares del año base (2010), considerando el mínimo de energía requerida por el poblador peruano [...]. Cada año se actualiza la canasta alimentaria con los precios medianos de los 110 productos que la conforman. Estos precios se obtienen para la población de referencia, por región natural y área de la Encuesta Nacional de Hogares. (INEI, 2016, p. 35) 
En cuanto al enfoque de la pobreza multidimensional, este se basa en los postulados de Amartya Sen, quien concibe el desarrollo como "un proceso de expansión de las libertades reales de las que disfrutan los individuos" (Sen, 2000, p. 3). De este modo, hay que ver a la pobreza como una cuestión de personas y sus capacidades y oportunidades en varias dimensiones, antes que de bienes y dinero. Con base en esto, la Oxford Poverty and Human Development Initiative (OPHI) ha desarrollado un Índice de Pobreza Multidimensional, el cual tiene en cuenta las dimensiones de educación, salud y condiciones de vida bajo determinadas ponderaciones (Alkire y Santos, 2010).

En el caso del Perú, tenemos que entre los trabajos que aplican este enfoque destaca el de Vásquez (2012), quien ajusta ponderaciones para medir la pobreza multidimensional con base en lo propuesto por Alkire y Foster (2011) como sigue:

Tabla 1

Componentes del índice de pobreza multidimensional

\begin{tabular}{c|c|l|c}
\hline Dimensión & Indicador & \multicolumn{1}{c|}{$\begin{array}{c}\text { La persona se considera pobre } \\
\text { (con privación) si: }\end{array}$} & $\begin{array}{c}\text { Peso } \\
\text { dentro } \\
\text { del IPM }\end{array}$ \\
\hline \multirow{5}{*}{ Educación } & $\begin{array}{c}\text { Escolaridad } \\
\text { familiar }\end{array}$ & $\begin{array}{l}\text { El jefe del hogar al que pertenece tiene } \\
\text { primaria completa o un nivel de educación } \\
\text { diferente. }\end{array}$ & $1 / 6$ \\
\cline { 2 - 5 } & $\begin{array}{c}\text { Matrícula } \\
\text { infantil }\end{array}$ & $\begin{array}{l}\text { El hogar donde vive tiene al menos un niño en } \\
\text { edad escolar (6-18) que no está matriculado (y } \\
\text { aún no termina la secundaria). }\end{array}$ & $1 / 6$ \\
\hline \multirow{5}{*}{ Salud } & $\begin{array}{c}\text { Asistencia } \\
\text { a centro de } \\
\text { salud }\end{array}$ & $\begin{array}{l}\text { Ante molestia, enfermedad o accidente; no } \\
\text { accede a los servicios de salud porque: no } \\
\text { tiene dinero, el centro de salud se encuentra } \\
\text { lejos de su vivienda o no tiene seguro de salud. }\end{array}$ & $1 / 6$ \\
\cline { 2 - 5 } & $\begin{array}{c}\text { Déficit } \\
\text { calórico }\end{array}$ & $\begin{array}{l}\text { No consume las calorías mínimas de acuerdo } \\
\text { a sus requerimientos. }\end{array}$ & $1 / 6$ \\
\cline { 2 - 5 } & Electricidad & Su vivienda no tiene electricidad. & $1 / 15$ \\
\cline { 2 - 5 } Condiciones & Agua & $\begin{array}{l}\text { Su vivienda no tiene acceso adecuado a agua } \\
\text { potable. }\end{array}$ & $1 / 15$ \\
\cline { 2 - 5 } & Desagüe & $\begin{array}{l}\text { Su vivienda no tiene desagüe con conexión a } \\
\text { red pública. }\end{array}$ & $1 / 15$ \\
\cline { 2 - 5 } & $\begin{array}{c}\text { Piso de la } \\
\text { vivienda }\end{array}$ & $\begin{array}{l}\text { El piso de su vivienda está sucio, con arena o } \\
\text { estiércol. }\end{array}$ & $1 / 15$ \\
\cline { 2 - 5 } & $\begin{array}{c}\text { Combustible } \\
\text { de cocina }\end{array}$ & $\begin{array}{l}\text { En su vivienda se usa generalmente carbón o } \\
\text { leña para cocinar. }\end{array}$ & $1 / 15$ \\
\hline
\end{tabular}

Fuente: Vásquez (2012) 
A partir de allí Vásquez realiza los cálculos de pobreza multidimensional para los años 2010 y 2011 con los datos de la ENAHO, y encuentra diferencias significativas en los resultados respecto del estándar de pobreza monetaria, con lo que se constituye el grupo de los "pobres no visibles para el Estado" (Vásquez, 2012). Asimismo, en una investigación posterior (Vásquez, 2013) aplica el enfoque de la pobreza multidimensional para evaluar los programas sociales del gobierno de Humala, encontrando serios problemas de cobertura y filtración.

Ahora, si la cuestión de definir y medir la pobreza es compleja, también lo es la de sus determinantes. Análogamente a lo que sucedía con el concepto de pobreza, puede decirse que hay un claro consenso respecto de que la pobreza es un fenómeno multicausal, es decir, determinado por múltiples causas. No obstante, el consenso se tiende a quebrar cuando se quiere especificar cuáles son los principales determinantes. Hay autores que ponen énfasis en las instituciones, otros en las variables monetarias, otros en el acceso a servicios, otros en las características sociodemográficas, etcétera. Pero en todo caso hay ciertos determinantes sobre cuya relevancia e interés hay suficiente consenso. Consideraremos cuatro de ellos.

Primero, un determinante clave de la pobreza es el acceso a servicios. Tanto el enfoque de pobreza multidimensional como el de necesidades básicas insatisfechas ponen marcado énfasis en esto. Tener acceso a agua potable, sistema de desagüe, electricidad, telefonía, etcétera es de suma importancia para reducir la vulnerabilidad de los hogares y la afectación al rendimiento escolar de los niños y a la productividad y empleabilidad de los padres.

Dado el contexto de lo precedente, el desarrollo de infraestructura es absolutamente necesario para la reducción de la pobreza. Siguiendo a Reinikka y Svensson (1999), podemos definir a la infraestructura como aquel capital complementario que ofrece los servicios de soporte necesarios para la operación de las actividades privadas. Así, un hogar que no tiene acceso a agua potable ni sistema de desagüe ni electricidad ni telefonía ve muy seriamente mermadas sus posibilidades de inserción en los mercados y, por tanto, de mejora económica.

Han sido varios los estudios que han analizado a la infraestructura como determinante de la pobreza. Destaca, por ejemplo, el estudio de Roy (2009), quien encontró una fuerte correlación negativa entre el Índice de Pobreza Humana y la infraestructura en la India para el periodo 1981-2001. Asimismo, Ogun (2010) encontró, por medio de un modelo VAR estructural, que el desarrollo de infraestructura permitió reducir la pobreza 
significativamente en las zonas urbanas de Nigeria durante el periodo 1970-2005.

En el caso peruano puede mencionarse el estudio de Torero, Escobal y Saavedra (2001), quienes encontraron impactos significativos de la infraestructura de agua potable, desagüe, electricidad y telefonía sobre la pobreza, siendo la tenencia de teléfono el factor más importante para las zonas urbanas. En esa línea, Webb (2013), luego de un amplio estudio, concluye que el motor central para el despegue rural en el Perú habría sido la transformación y mejora de la plataforma comunicativa, incluyendo la llegada y rápida propagación de las telecomunicaciones. A su vez, Aparicio, Jaramillo y San Román (2011), tomando también la infraestructura de agua, desagüe, electricidad y teléfono, hallan mediante análisis de corte transversal, que "la probabilidad de ser pobre pasa de $45 \%$ cuando no se tiene infraestructura alguna en el hogar a $12 \%$ cuando se tienen las cuatro infraestructuras" (p. 41).

En segundo lugar, tenemos los derechos de propiedad. Y es que no es solamente que los pobres carecen de ingreso sino que también carecen de activos para generar ingresos (y, por ende, sustentar su consumo), estando esto último estrechamente relacionado con los derechos de propiedad (Meinzen-Dick, 2009). En esa línea, Sen (1988) ha sostenido que hay una relación estrecha entre pobreza y hambre, siendo que las causas del hambre dependerían materialmente de cómo están estructurados los derechos de propiedad.

En cuanto a estudios empíricos al respecto, destaca el de Norton (1998), quien realiza un análisis cruzado entre países y halla que "los derechos de propiedad fuertes tienden a reducir la privación de las personas más pobres del mundo mientras que derechos de propiedad débiles tienden a agravar la privación de las personas más pobres del mundo" (p. 244).

Para el caso peruano tenemos el estudio de De Soto (1986), quien sostiene que la pobreza se debe fundamentalmente a la falta de acceso a la propiedad formal, la cual, a su vez, dificulta el acceso al crédito y a mercados más amplios. A su vez, tenemos el estudio de Bandeira, Sumpsi y Falconi (2009), quienes analizan el impacto de la titulación de la tierra sobre la reducción de la pobreza en las áreas rurales del Perú (junto con Honduras) y llegan a establecer que la titulación "es necesaria y suficiente para aumentar ligeramente la actividad de los mercados de alquiler de tierra y de crédito, con el consiguiente impacto, pequeño pero significativo, sobre los ingresos de los hogares" (p. 33). 
En tercera instancia tenemos que la educación o, más exactamente, el carecer de ella es un determinante de la pobreza. La educación de los niños es definitivamente un factor crucial para que un hogar pueda salir de la pobreza en el futuro y, a su vez, la educación de los jefes de hogar es determinante para que pueda hacerlo (o ya lo haya hecho) en el presente. Desde el trabajo de Becker (1964) se tiene como establecido que la inversión en "capital humano" es clave para el progreso económico, tanto a nivel de naciones como de individuos, y en ello el aspecto primordial es la educación.

Así encontramos que en el estudio de Attanasio y Székely (2001), quienes analizan la pobreza en términos de activos, se considera que el primer activo es el "capital humano" y se señala que la medida más usada del mismo son los años de educación formal. En ese contexto, estos autores encontraron que en América Latina:

tener primaria incompleta implica, en promedio, un ingreso 18\% más alto que el de las personas sin ninguna educación. La educación primaria completa da un retorno de $37 \%$, mientras que la educación secundaria incompleta y completa tiene retornos del $61 \%$ y $95 \%$, respectivamente. Los retornos más altos se observan para la educación superior, con 152\% en promedio. (p. 22)

En el caso peruano puede mencionarse el estudio de Benavides (2004), donde se encuentra que, aunque hay diversos condicionantes sociales que influyen, la educación aumenta las probabilidades de movilidad ascendente y tiene un importante impacto intergeneracional en el sentido de que, en general, a mayor educación de los padres se halla un mayor nivel de educación de los hijos.

Finalmente está el factor salud. Es claro que la salud constituye un determinante de la pobreza pues un mal estado de salud no solo afecta a la distribución de los gastos en el hogar y al rendimiento escolar de los niños sino también a factores estrechamente relacionados con la capacidad de generar ingresos, como la productividad, continuidad y disponibilidad laboral.

En cuanto a estudios empíricos en esta línea puede mencionarse el de Gupta y Mitra (2004), quienes analizaron la relación entre crecimiento, salud y pobreza usando un panel de datos para los estados de la India y hallaron que "a pesar de que el crecimiento tiende a reducir la pobreza, mejoras sustanciales en el estado de salud son también necesarias para reducir la pobreza" (p. 193). Asimismo, realizaron estimaciones sobre las 
pérdidas en PIB y productividad por causa de la malnutrición y encontraron, por ejemplo, que en algunos países no desarrollados la anemia por deficiencia de hierro por sí sola puede implicar una pérdida de entre $2 \%$ a $8 \%$ del PIB.

En el caso peruano tenemos el estudio de Valdez et al. (2013) donde, entre otros factores, se analiza la relación entre salud y pobreza con especial atención en los condicionantes del acceso a sistemas de aseguramiento como el Sistema Integral de Salud (SIS) o el Seguro Social de Salud del Perú (EsSalud), siendo que se encuentran relevantes problemas de subcobertura, exclusión y desigualdad. A su vez, en lo que se refiere a la estimación de la influencia sobre la productividad, un importante antecedente es el estudio de Cortez (1999) quien, analizando por género y región en el Perú, halla que el indicador de salud tiene un efecto positivo y significativo sobre la productividad.

Dado todo esto, la propuesta del presente trabajo es crear un "puente" entre el enfoque de pobreza monetaria y el de pobreza multidimensional considerando a esta última en términos de sus dimensiones determinantes de la misma explicadas previamente.

\section{METODOLOGÍA}

Una discusión metodológica frecuente en los estudios sobre pobreza consiste en decidir si tomar el enfoque de la pobreza monetaria o el de la multidimensional. En ese contexto se suelen plantear críticas al enfoque de la pobreza monetaria por ser muy limitado y se aboga por el enfoque de la pobreza multidimensional por ser más amplio. Sin embargo, si lo que nos interesa específicamente es estudiar los determinantes de la pobreza tanto cualitativa como cuantitativamente, hacerlo a través del índice multidimensional de pobreza puede llevar a redundancias, pues buena parte de los componentes de estos índices no son solamente dimensiones de la pobreza sino también sus causas.

Consideremos por ejemplo el primer indicador del esquema multidimensional de Vásquez (2012): la escolaridad familiar medida en términos de nivel educativo del jefe del hogar, a la que se asigna una ponderación de 1/6. Es claro que el nivel educativo del jefe del hogar es un determinante relevante respecto de la situación de pobreza o no del hogar al que pertenece. Por tanto, resulta de interés no solo analizar esta relación causal sino también cuantificarla de modo específico. Pero si utilizamos como variable para captar la pobreza el índice de pobreza multidimensional, 
obtendremos una redundancia al cuantificar la relación dado que ya en el mismo índice se impone una ponderación predeterminada respecto del nivel educativo del jefe del hogar.

De este modo, lo que se puede hacer es analizar la evolución del índice de pobreza multidimensional y de sus componentes de forma desagregada en términos de gráficas, estadísticas descriptivas y comparaciones, tanto transversales como longitudinales, como se suele encontrar en la literatura de ese enfoque, pero no propiamente estimar relaciones econométricas específicas de modo consistente y válido, al menos para aquellas variables causales que sean también componentes del índice.

Por lo anterior, resulta necesario tomar una medida de la pobreza que no incorpore directamente componentes que sean a la vez sus determinantes. La medición desde el enfoque de la pobreza monetaria cumple con esta condición.

Es cierto que, como se mencionó, el enfoque de la pobreza monetaria ha recibido diversas críticas. Tiene, en efecto, limitaciones, pero no necesariamente lo invalidan. Al contrario, se trata de un enfoque útil en el contexto de los estudios que aplican ajustes econométricos, además de que es todavía el más usado en la medición de la pobreza por instituciones nacionales e internacionales.

Adicionalmente, es importante considerar también las matizaciones respecto de las críticas al enfoque de pobreza monetaria a la luz de estudios para el caso peruano. Por ejemplo, Adrianzén (2013) halla que, si analizamos en términos del número de habitantes, es del todo claro que la pobreza se concentra en Lima. A su vez, Vásquez (2013), quien es crítico del enfoque monetario y aboga por el multidimensional, encuentra de todos modos que "ambos enfoques muestran resultados similares en Lima Metropolitana" (p. 33). En consecuencia tenemos que la medición por medio de la pobreza monetaria no discrepa mayormente de la multidimensional para la zona que concentra la mayor cantidad de pobres del país.

Por tanto, es claro que las mediciones en términos de pobreza monetaria, a pesar de tener limitaciones, pueden ser válidamente usadas, siendo que, al menos para el caso peruano, tenemos importantes atenuantes respecto de las críticas que se les hacen. Así, siguiendo el criterio del INEI (2016), se considerará como pobres monetarios a las personas que residen en hogares cuyo gasto per cápita es insuficiente para adquirir una canasta básica.

Dado esto, la variable dependiente de nuestro modelo será la pobreza monetaria expresada en términos binarios respecto de si se presenta o no 
la condición de pobre monetario (" 1 " si la tiene, " 0 " en caso contrario), considerando para ello el gasto per cápita de los hogares frente a la línea de pobreza dada por el costo de la canasta básica de consumo.

Luego, en lo que respecta a las variables independientes que serían los determinantes de la pobreza, aunque sabemos que podrían tomarse varias más pero al mismo tiempo enfatizando ciertas relaciones específicas, las tomaremos en correspondencia con los cuatro determinantes base descritos en el apartado precedente y seleccionando aquellas que consideramos más relevantes en el marco de los datos disponibles.

En cuanto al acceso a servicios, se consideró como más relevante estudiar el acceso a los de agua potable, electricidad y telefonía, respecto de cuya importancia hay un claro consenso en la literatura. Se tomarán estas variables de modo binario: " 1 " si se tiene el servicio en cuestión y " 0 " en caso contrario.

Respecto de los derechos de propiedad, la variable que se tomará será si el jefe del hogar tiene la propiedad de la vivienda en que habita o no. También aquí se usará una estructura binaria: "1" si es propietario de su vivienda y "0" si no lo es.

En cuanto a la educación, se considerará si el jefe del hogar tiene educación primaria completa y también si tiene educación secundaria completa. Es cierto que el acceso a escolaridad de los hijos es también una dimensión relevante de la pobreza pero tomamos el nivel educativo del jefe del hogar y no el de los hijos porque estamos estudiando los determinantes de la pobreza y es el nivel educativo del primero y no el de los segundos lo que actúa como factor causal al respecto. La variable será tomada en términos binarios: "1" si el jefe del hogar tiene completo el nivel de educación correspondiente y " 0 " en caso contrario.

Finalmente, en lo que se refiere a salud se considerarán como variables si la persona cuenta con seguro de salud, considerando por separado el seguro EsSalud, que cubre fundamentalmente a la población asalariada y el SIS, que subsidia la provisión de servicios de salud para las familias en situación de pobreza. Se busca captar con ello el efecto diferencial del sistema de aseguramiento con aportaciones y aquel cuya modalidad predominante es la gratuita. Como en los casos anteriores, se considerarán estas variables en términos binarios: "1" si se cuenta con el sistema de seguro en cuestión y " 0 " en caso contrario.

El resumen esquemático de las variables se muestra en la tabla 2. 
Tabla 2

Variables del modelo

\begin{tabular}{|c|c|c|}
\hline Determinante & Variable & Descripción \\
\hline & Pobre & $\begin{array}{l}1=\text { hogar pobre } \\
0=\text { contrario }\end{array}$ \\
\hline \multirow{3}{*}{ Acceso a servicios } & Agua & $\begin{array}{l}1=\text { tiene acceso a servicio de agua potable } \\
0=\text { caso contrario }\end{array}$ \\
\hline & Electricidad & $\begin{array}{l}1=\text { tiene acceso a servicio de electricidad } \\
0=\text { caso contrario }\end{array}$ \\
\hline & Telefonía & $\begin{array}{l}1=\text { tiene acceso a servicio de telefonía } \\
0=\text { caso contrario }\end{array}$ \\
\hline $\begin{array}{l}\text { Derechos de } \\
\text { propiedad }\end{array}$ & $\begin{array}{l}\text { Propiedad } \\
\text { de vivienda }\end{array}$ & $\begin{array}{l}1=\text { es propietario de la vivienda en que habita } \\
0=\text { caso contrario }\end{array}$ \\
\hline \multirow{2}{*}{ Educación } & $\begin{array}{l}\text { Educación } \\
\text { primaria }\end{array}$ & $\begin{array}{l}1=\text { tiene educación primaria completa } \\
0=\text { caso contrario }\end{array}$ \\
\hline & $\begin{array}{l}\text { Educación } \\
\text { secundaria }\end{array}$ & $\begin{array}{l}1=\text { tiene educación secundaria completa } \\
0=\text { caso contrario }\end{array}$ \\
\hline \multirow{2}{*}{ Salud } & Seguro EsSalud & $\begin{array}{l}1=\text { se encuentra afiliado a EsSalud } \\
0=\text { caso contrario }\end{array}$ \\
\hline & Afiliación al SIS & $\begin{array}{l}1=\text { encuentra afiliado al SIS } \\
0=\text { caso contrario }\end{array}$ \\
\hline
\end{tabular}

Elaboración propia

Todos los datos para las variables se obtienen de la Encuesta Nacional de Hogares (ENAHO) del INEI. En términos espaciales el análisis abarca todo el Perú y, en términos temporales, los periodos 2009-2010 y 2014-2015, correspondientes a los dos últimos años completos de los dos gobiernos precedentes, siendo que los años 2011 y 2016 serían periodos de transición (cambio de gobierno). Ha de anotarse respecto de las observaciones, que se han tomado todos los datos que quedaron luego de excluir aquel conjunto que implicaba algún problema de missing data (datos perdidos), lo cual es común en los datos de la ENAHO por diversas causas (errores de encuesta, de registro, etcétera). En todo caso, queda una gran cantidad de observaciones para cada uno de los años en estudio: 16218 para el 2009, 16294 para el 2010, 24093 para el 2014 y 24838 para el 2015. Tal cantidad de observaciones permite que los resultados sean sólidos.

En cuanto a cómo establecer las relaciones entre estas variables, tenemos que tanto la variable dependiente como las independientes son cualitativas, por lo que se buscará construir un "puente" entre lo cualitativo y lo cuantitativo, entre la pobreza monetaria y la multidimensional. 
El tipo de modelo econométrico que se ajusta a ello es logístico. De este modelo se obtendrán los efectos marginales que nos darán la medida de la probabilidad de ser pobre monetario para cada variable considerada. Por supuesto, como sucede con toda elección metodológica, usar un modelo logit tiene limitaciones pero es el que resulta más conveniente para el tipo de análisis que aquí queremos realizar en términos de comparaciones entre periodos. Además, este tipo de aproximación econométrica tiene antecedentes en diversos estudios sobre pobreza en el Perú (Chumpitaz y Jara, 2008; Cavero, 2015; Herrera y Cozzubo, 2016).

\section{RESULTADOS}

Conforme a la metodología establecida en el apartado precedente, se corrieron modelos logit con base en los datos de la ENAHO para los años 2009, 2010, 2014 y 2015, es decir, los dos últimos años completos de los dos gobiernos anteriores. En las tablas 3 y 4 se muestran los resultados obtenidos.

En términos generales vemos que las variables independientes, consideradas en conjunto, contribuyen significativamente a explicar la condición de pobreza en cada uno de los periodos en estudio. En efecto, para cada caso se halla que el p-valor del test de la razón de verosimilitud aparece como nulo, de modo que el modelo sería conjuntamente significativo incluso a un nivel de confianza del $99 \%$. Asimismo, tenemos que los resultados son robustos dado que se han obtenido a partir de una gran cantidad de observaciones.

En cuanto a resultados específicos, hay varios que son relevantes. Los analizaremos en términos de los grupos de determinantes de la pobreza demarcados previamente.

Primero, respecto del acceso a servicios, tenemos que aparece claramente como el determinante más relevante para la reducción de la pobreza. En efecto, para todos los años en estudio los coeficientes para agua potable, electricidad y telefonía aparecen con signo negativo y todos ellos son muy significativos individualmente. Estos resultados coinciden básicamente, sobre todo en lo referido a electricidad y telefonía, con los hallados por Aparicio et al. (2011). 


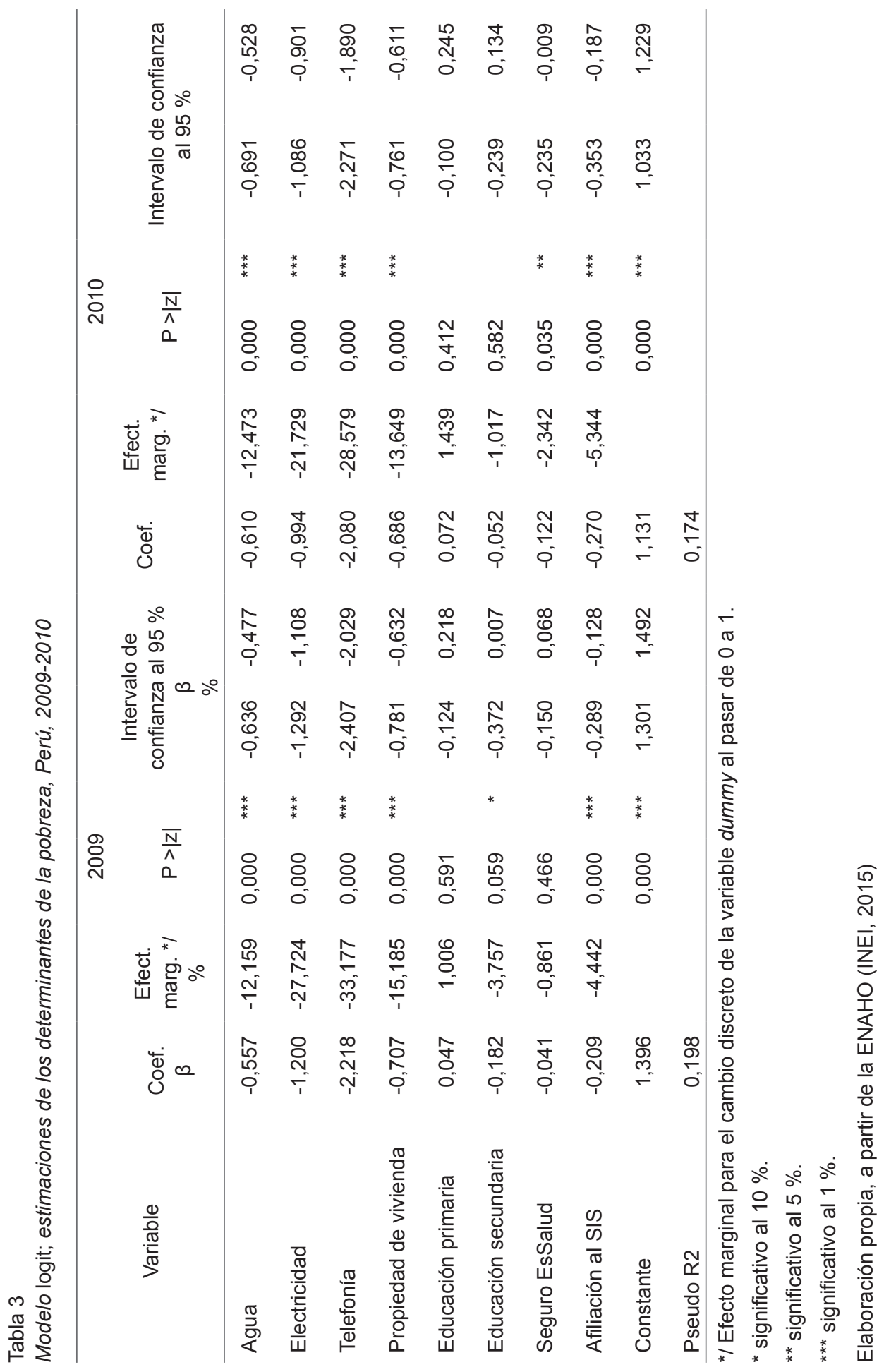




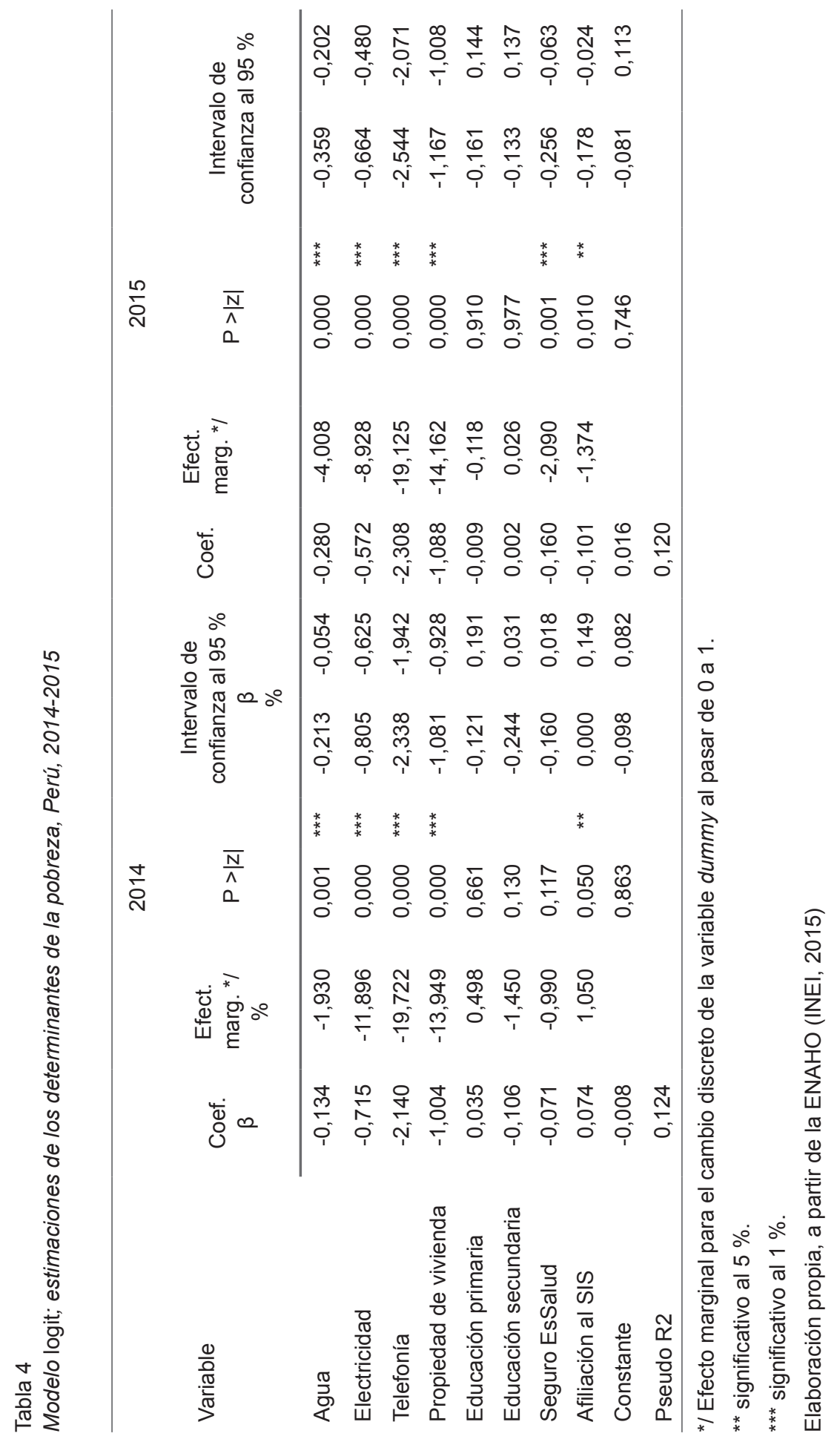


Respecto de la evolución de la influencia de estas variables, se encuentra que mientras en el periodo 2009-2010 el acceso a agua potable reducía la probabilidad de ser pobre en alrededor de un $12 \%$, para el 2014 la reduce en un $2 \%$ y para el 2015 en un $4 \%$. Esta perceptible reducción de la incidencia del acceso a agua potable sobre la pobreza puede explicarse por cuanto en el periodo inicial había una marcada presencia del problema de falta de acceso a este servicio, especialmente en las zonas rurales (Aparicio et al., 2011). Sin embargo, luego de ello ha habido importantes avances; ya hacia el 2012, alrededor de 1541153 personas dejaron de tener privación respecto del acceso a agua potable (Vásquez, 2013), y a esto hay que sumar lo logrado por programas como Agua para Todos y el Programa Nacional de Agua y Saneamiento Rural. Con ello, para el periodo 20142015 el problema está presente en una medida mucho menor y el efecto marginal de las mejoras es menor.

Algo similar sucede en cuanto al acceso a electricidad. Mientras en el 2009 y el 2010 el acceso a este servicio reducía la probabilidad de ser pobre en 27,72 y $21,73 \%$, respectivamente, para el 2014 la reduce en $11,90 \%$ y para el 2015 en 8,93 \%. Esta menor incidencia de la variable puede explicarse por cuanto la infraestructura eléctrica ha ido cubriendo cada vez más zonas del país.

En cuanto al acceso a telefonía es de notar que se trata claramente de la variable que más influye en reducir la probabilidad de estar en condición de pobreza. En efecto, en el 2009, tener acceso a telefonía reducía la probabilidad de ser pobre en un 33,18 \% y en el 2010 en un 28,58 \%; en el periodo 2014-2015, si bien la incidencia disminuye, pasando a estar en torno al $19 \%$, sigue siendo no solo alta sino la más alta de todas las variables incluidas en el modelo. Esta fuerte incidencia del acceso a telefonía en la reducción de la pobreza coincide con lo hallado por Aparicio et al. (2011) y también con el estudio de Apoyo Consultoría (2010), en el que se encuentra que hay un efecto positivo y robusto de la tenencia de teléfonos móviles en el ingreso de los hogares de las zonas rurales del Perú, siendo que la introducción de un teléfono móvil permitía incrementar en alrededor de S/ 900 el ingreso anual. Ello es coherente pues el teléfono móvil permite múltiples beneficios en las actividades económicas, como mejor coordinación con proveedores, reducción de los costos de transporte, más eficiente organización interna del trabajo, mayor contacto con clientes, acceso a nuevos mercados, etcétera.

En segunda instancia tenemos el determinante de derechos de propiedad, respecto del cual se consideró como variable si el jefe del hogar es propietario de la vivienda en que habita o no. A diferencia de lo que 
sucedía con el acceso a servicios, la incidencia de la propiedad de la vivienda se ha mantenido prácticamente igual en los periodos estudiados. En el 2009 la propiedad de la vivienda reducía la probabilidad de ser pobre en un 15 \% y en los años 2010, 2014 y 2015 la reduce en torno a un 14 \%. La razón de ello puede encontrarse en que la falta de acceso a la propiedad inmobiliaria es un problema que todavía persiste, de modo que ser propietario aún sería un factor diferencial relevante. Y es que si bien se ha dado una muy considerable expansión del sector inmobiliario peruano en los últimos años, ello no se ha correspondido con un aumento proporcional en el acceso a propiedad de las viviendas por parte de las personas de bajos recursos pues, como se ha señalado en un informe del Bank for International Settlement (Scatigna, Szemere y Tsatsaronis, 2014), el crecimiento de precios inmobiliarios en el Perú ha sido el mayor del mundo entre el 2008 y el 2014, en un contexto en que, mientras el $70 \%$ de la oferta de viviendas se ubicaba sobre los USD 80 000, ya no se evidenciaba una mayor demanda por encima de ese precio (Perea, Grippa, Crispin y Sánchez, 2015), cuando previamente se había hallado que el $90 \%$ de los peruanos que constituían la "demanda insatisfecha" no disponía de más de USD 30000 para vivienda (Westreicher, 2013).

En tercera instancia tenemos el factor educación. En este caso se encuentra que tener educación primaria completa o secundaria completa tiene un efecto ambiguo o muy pequeño en reducir la probabilidad de ser pobre. Así, en el 2009, tener educación primaria completa aumenta la probabilidad de ser pobre en 1,01 \% y tener secundaria completa la reduce en 3,76 \%; en el 2010, tener primaria completa aumentaría esta probabilidad en $1 \%$ y tener secundaria completa la reduciría en $1 \%$; en el 2014, tener primaria completa la aumentaría 0,5 \% y tener secundaria completa la reduciría en $1,5 \%$, y en el 2015, tener primaria completa la reduciría en 0,12 \% y tener secundaria completa la aumentaría en 0,03 \%. Por supuesto que llama la atención encontrar que en varios casos el signo no es el esperado, lo que sería especialmente problemático si los coeficientes respectivos fueran individualmente significativos. Pero, más bien, encontramos que ninguno de los coeficientes relacionados con educación es individualmente significativo, ni siquiera al $10 \%$. Adicionalmente tenemos que en los intervalos de confianza al $95 \%$, la cota inferior del intervalo para el coeficiente y el efecto marginal tiene signo negativo en todos los casos. Por tanto, todos los coeficientes de signo distinto al esperado son bajos, no significativos y pueden tener el signo esperado dentro de sus intervalos de confianza.

Ahora bien, el hecho de que los coeficientes asociados a la educación primaria y secundaria no sean estadísticamente significativos en general 
es algo que no debe soslayarse, pues tiene un significado relevante en el ámbito empírico. Y es que ello implicaría que tener educación primaria o secundaria completa no haría realmente la diferencia para salir de la condición de pobreza. Es decir, es casi seguro que no tener educación alguna mantendrá a alguien en la pobreza pero, al mismo tiempo, tener educación primaria o secundaria no solo no garantiza sino que no influye decisivamente en la reducción de la probabilidad de ser pobre.

La razón de esto puede hallarse en el nuevo contexto del mercado laboral. En efecto, siendo el subempleo algo que caracteriza al mercado de trabajo peruano, pueden encontrarse personas incluso con educación universitaria completa que tienen trabajos mal remunerados y sustancialmente por debajo de su nivel de competencias profesionales. Como reportan Yamada, Lavado y Martínez (2014): "A pesar del elevado crecimiento de la economía peruana en los últimos diez años, los profesionales universitarios se enfrentan a dificultades cada vez mayores para desempeñarse en ocupaciones laborales acorde [sic] a su formación educativa superior, habilidades e inversiones educativas" (p. 2). En este contexto es difícil que la educación primaria o secundaria vaya a hacer la diferencia en el acceso a mejores trabajos. Ello podía ocurrir incluso hasta mediados del siglo pasado, pero en la economía actual hay más especialización y sofisticación, no solo de los trabajos altamente remunerados sino incluso de los medianamente remunerados. Por tanto, a personas que solo tienen primaria o secundaria completa les queda la alternativa de acceder a trabajos mal remunerados, principalmente en el sector informal.

Finalmente, respecto de la salud se encuentra que tener seguro de salud reduce la probabilidad de ser pobre pero de modo marginal. En el 2009, contar con seguro EsSalud reducía la probabilidad de ser pobre en 0,9\% y contar con seguro SIS lo hacía en 4,4 \%. En el 2010 estas probabilidades eran de 2,4 \% y 5,3\%, respectivamente. En el 2014 se encuentra que tener seguro EsSalud reduce la probabilidad de ser pobre en $1 \%$ y tener seguro SIS la aumenta en $1 \%$. Por último, en el 2015 se halla que estar afiliado a EsSalud reduce la probabilidad de ser pobre en 2,1 \% y estar afiliado al SIS hace lo propio en un 1,4\%. El único caso en que el coeficiente no tiene el signo esperado es la afiliación al SIS en el 2014. Sin embargo, este coeficiente está justo en el límite de no ser significativo al $5 \%$.

En la explicación de la baja incidencia del acceso a seguro de salud en la reducción de la probabilidad de ser pobre debe tenerse en consideración que los datos de los que se dispone son sobre la cantidad de personas y hogares que están asegurados, pero no informan de por sí sobre la calidad 
de la atención. De este modo, si para que alguien logre ser atendido tiene que hacer varios trámites y esperar incluso por meses, no se puede esperar que el hecho de tener seguro contribuya de modo consistente y sustancial a la mejora de las condiciones de vida para salir o no caer en situación de pobreza. De acuerdo con lo reportado por el Latinobarómetro, solo el $31,6 \%$ de la población peruana está satisfecha con la atención que recibe en los hospitales públicos (Lazo-Gonzales, Alcalde-Rabanal y EspinosaHenao, 2016, p. 68).

Por otro lado, cabe mencionar los problemas de focalización de los programas que terminan mermando su propia efectividad. Así, ya Vásquez (2012) señalaba, con base en datos del 2011, que 21000 personas estaban recibiendo los beneficios tanto del seguro SIS como de EsSalud, lo cual es incompatible, pues quien está asegurado en EsSalud ya no puede ser parte de la población objetivo del SIS y que, entre ellos, el $68 \%$ no eran pobres multidimensionales. Este tipo de filtraciones en el SIS pueden ser, en cierta medida, una consecuencia indeseada de la eliminación de restricciones administrativas realizada para aumentar la cobertura.

\section{CONCLUSIONES}

Los resultados obtenidos en esta investigación muestran varios aspectos interesantes. Se encuentra que el acceso a servicios es el determinante más importante de la pobreza. Así, tener agua, electricidad y teléfono reducen considerablemente la probabilidad de estar en condición de pobreza, siendo claramente el acceso a telefonía el factor de mayor relevancia. En ese contexto, los esfuerzos públicos deben focalizarse en extender e impulsar el desarrollo de la infraestructura de los servicios de agua, electricidad y telefonía de modo tal que lleguen a todas las zonas del país. En ello podrían tener un rol muy importante las asociaciones público-privadas.

En cuanto a los derechos de propiedad, tener propiedad de la vivienda en que se habita reduce la probabilidad de ser pobre en un $14 \%$ o $15 \%$. Es de notar que esta probabilidad se ha mantenido casi constante, lo cual parece deberse a que, si bien ha habido una considerable expansión inmobiliaria en los últimos años, los precios han estado muy por encima de lo que puede financiar la mayoría de los peruanos. Por tanto, se requiere impulsar alternativas sostenibles para que las personas de bajos recursos puedan acceder a casa propia, mejorando y extendiendo programas como Mivivienda y Techo Propio. 
Respecto de la educación es interesante notar que tener primaria o secundaria completa no tiene en general una incidencia significativa en reducir la probabilidad de ser pobre. Ello puede deberse a las nuevas características del mercado laboral, donde se requieren conocimientos especializados incluso para trabajos medianamente remunerados y se observa un extendido subempleo incluso de egresados universitarios. Así, el solo hecho de tener educación primaria o secundaria difícilmente puede hacer una diferencia sustancial en la generación de ingresos. En ese sentido, una alternativa sería diseñar políticas educativas orientadas a mejorar la calidad y la cantidad de la oferta de profesionales técnicos.

En lo referido a salud se encuentra que tener seguro reduce la probabilidad de ser pobre de modo distinguible pero poco significativo, hallándose la mayor incidencia en el 2010, cuando tener seguro EsSalud reducía la probabilidad de ser pobre en 2,4\% y estar afiliado al SIS la reducía en $5,3 \%$. Esta comparativamente baja incidencia puede deberse a deficiencias de calidad en la atención y a problemas de focalización. Por tanto, se requiere diseñar políticas o sistemas de control específicos respecto de esos problemas.

Estos son los principales hallazgos del presente trabajo en que se ha analizado la pobreza monetaria desde diferentes determinantes relacionados con la pobreza multidimensional. Por supuesto, este análisis es general para el Perú, de modo que una línea de investigación futura podría ser la desagregación en términos de capital y provincias, zonas rurales y urbanas o incluso regiones, captándose así dinámicas más específicas de la pobreza y sus determinantes. En ese contexto, nuestro trabajo constituye una base para ampliar la investigación desde la perspectiva del uso de modelos de respuesta cualitativa para relacionar el enfoque de pobreza monetaria (cuantitativo) con el de pobreza multidimensional (cualitativo).

\section{REFERENCIAS}

Adrianzén, C. (2013). Crecimiento y pobreza en el Perú: 2001-2011. Revista de Economía y Derecho, 10(37), 35-66.

Alkire, S. y Santos, M. (2010). Acute Multidimentional Poverty: A New Index for Developing Countries. Oxford Poverty and Human Development Initiative, Working Paper n. ${ }^{\circ} 38$.

Alkire, S. y Foster, J. (2011). Counting and multidimentional poverty measurement. Journal of Public Economics, 95(7), 476-487. 
Aparicio, C., Jaramillo, M. y San Román, C. (2011). Desarrollo de la infraestructura y reducción de la pobreza: El caso peruano. Lima: Consorcio de Investigación Económica y Social.

Apoyo Consultoría (2010). El impacto de las telecomunicaciones en el desarrollo: El caso de la telefonía móvil en el ámbito rural. Informe, Lima: Apoyo.

Attanasio, O. y Székely, M. (2001). Going beyond income: Redefining poverty in Latin America. En: Attanasio, O. y Székely, M. (eds.). Potrait of the Poor: An Assets-Based Approach. Washington D. C.: InterAmerican Development Bank.

Bandeira, P., Sumpsi, J. y Falconi, C. (2009). Los efectos de la formalización de la propiedad de la tierra sobre la reducción de la pobreza rural: Evidencia de Perú y Honduras. Estudios Económicos de Desarrollo Internacional, 9(1), 33-58.

Becker, G. (1964). Human Capital: A Theoretical and Empirical Analysis, with Special Reference to Education. Nueva York: National Bureau of Economic Research.

Benavides, M. (2004). Educación y estructura social en el Perú: Un estudio acerca del acceso a la educación superior y la movilidad intergeneracional en una muestra de trabajadores urbanos. En: Arregui, P. (ed.). Es posible mejorar la educación peruana: Evidencias y posibilidades. Lima: Grupo de Análisis para el Desarrollo.

Cavero, O. (2015). La dinámica de la pobreza en el Perú (2004-2011): Un análisis de las transiciones y sus determinantes. Lima: Pontificia Universidad Católica del Perú (tesis de maestría).

Chumpitaz, A. y Jara, C. (2008). Dinámicas de la pobreza extrema y no extrema: análisis para el caso peruano (2003-2006). Apuntes (63), 79-106.

Cortez, R. (1999). Salud y productividad en el Perú: Un análisis empírico por género y región. Banco Interamericano de Desarrollo, Documento de trabajo R-363.

De Soto, H. (1986). El otro sendero. Lima: Instituto Libertad y Democracia.

Gupta, I. y Mitra, A. (2004). Economic growth, health and poverty: An exploratory study for India. Development Policy Review, 22(2), 193-206.

Herrera, J. y Cozzubo, A. (2016). La vulnerabilidad de los hogares a la pobreza en el Perú, 2004-2014. Développement, Institutions et Mondialisation, Document de Travail. 
Instituto Nacional de Estadística e Informática (2016). Encuesta Nacional de Hogares (ENAHO 2009-2015). Lima: INEI.

Instituto Nacional de Estadística e Informática (2016). Evolución de la pobreza monetaria 2009-2015. Lima: INEI, Informe técnico.

Lazo-Gonzales, O., Alcalde-Rabanal, J. y Espinosa-Henao, O. (2016). El sistema de salud en Perú: Situación y desafíos. Lima: Colegio Médico del Perú.

Meinzen-Dick, R. (2009). Property rights for poverty reduction? United Nations, Department of Economic Affairs, Working Paper n. ${ }^{0} 91$.

Norton, S. (1998). Poverty, property rights and human well-being: A crossnational study. Cato Journal, 18(2), 233-245.

Ogun, T. (2010). Infrastructure and poverty reduction: Implications for urban development in Nigeria. United Nations University/World Institute for Development Economics Research, Working Paper n. ${ }^{o}$ 2010/43.

Perea, H., Grippa, F., Crispin, Y. y Sánchez, R. (2015). Perú: Situación inmobiliaria 2014. Lima: BBVA Research, Informe.

Ravallion, M. (2015). The World Bank's new global poverty line. Center for Global Development. Recuperado de http://www.cgdev.org/blog/ world-bank\%E2\%80\%99s-new-global-poverty-line

Reinikka, R. y Svensson, J. (1999). How inadequate provision of public infrastructure and services affect private investment. World Bank, Working Paper n. ${ }^{\circ} 2262$.

Roy, K. (2009). Effect of public infrastructure on poverty reduction in India: A State level study for the period 1981-2001. Indian Journal of Millenium Developmet Studies, 4(1), 99-111.

Scatigna, M., Szemere, R. y Tsatsaronis, K. (2014). Residential property price statistics across the globe. BIS Quaterly Review (septiembre).

Sen, A. (1988). Property and hunger. Economics and Philosophy, 4(1), 57-68.

Sen, A. (2000). Desarrollo y libertad. Buenos Aires: Planeta.

Torero, M., Escobal, J. y Saavedra, J. (2001). Distribution, access and complementarity: Capital of the poor in Peru. En: Attanasio, O. y Székely, M. (eds.). Potrait of the Poor: An Assets-Based Approach. Washington D. C.: Inter-American Development Bank. 
Valdez, W., Napanga, E., Oyola, A., Mariños, J., Vílchez, A., Medina, J. y Berto, M. (2013). Análisis de la situación de salud en el Perú. Lima: Ministerio de Salud.

Vásquez, E. (2012). El Perú de los pobres no visibles para el Estado: La inclusión social pendiente a julio del 2012. Lima: Centro de Investigaciones de la Universidad del Pacífico, documento de discusión.

Vásquez, E. (2013). Las políticas y programas sociales del gobierno de Ollanta Humala desde la perspectiva de la pobreza multidimensional. Lima: Centro de Investigaciones de la Universidad del Pacífico, documento de discusión.

Webb, R. (2013). Conexión y despegue rural. Lima: Universidad San Martín de Porres.

Westreicher, G. (2013). El 90 \% de la demanda inmobiliaria 'insatisfecha' no puede pagar más de US\$ 30 mil por vivienda. Diario Gestión, 6 de junio.

Yamada, G., Lavado, P. y Martínez, J. (2014). ¿Una promesa incumplida?: La calidad de la educación superior universitaria y el subempleo profesional en el Perú. Lima: Centro de Investigaciones de la Universidad del Pacífico, documento de discusión. 